\title{
Systematic Renormalization at all Orders in the DiffRen and Improved Epstein-Glaser Schemes
}

\author{
José M. Gracia-Bondía
}

\begin{abstract}
Proceeding by way of examples, we update the combinatorics of the treatment of Feynman diagrams with subdivergences in differential renormalization from more recent viewpoints in Epstein-Glaser renormalization in $x$-space.
\end{abstract}

\section{Introduction}

Ultraviolet divergent amplitudes of Feynman diagrams in $x$-space are given by functional expressions too singular at short distances to define distributions on all of space(-time). Quite often, however, they can be represented on their domain of definition as derivatives of bona fide distributions, and so extended to the whole space. "Differential renormalization" (DiffRen for short) in this sense does not need previous regularization steps. It was introduced by Freedman, Johnson and Latorre in a path-breaking article [1.

While that reference is a true tour de force, showing the calculational power of the method (and of its authors), it did not attempt to disentangle the combinatorics of successive renormalizations for non-primitive graphs in a systematic way. This task was taken up in later work in differential renormalization [2].

Differential renormalization remained popular during the nineties, to peter out in the third millennium. Now, a spate of papers [3 [5] have recently dealt with renormalization in $x$-space in the spirit of the classic article by Epstein and Glaser [6], whose kinship with DiffRen is evident. Each of those solves the renormalization recursion in its own way. Among them, reference [4] adheres to DiffRen closely and improves it. Our aim here is to pedagogically revisit from its viewpoint the combinatorics of the "subtraction" of subdivergences in 2] as well, comparing methods and (hopefully) bringing improvement again. 
As in the last-mentioned paper, for simplicity we deal mostly with the Euclidean massless $\phi_{4}^{4}$ model.

\section{The importance of degree}

We start by a point of rigour, that some readers may wish to skip. Naturally the first step in [2] is to determine whether a given graph needs renormalization. The answer they give, in view of Weinberg's "power counting" theorem [7, 8, is to find the superficial degree of each generalized vertex (subgraph) in the graph. Now, that theorem yields a sufficient condition for renormalizability. While the question of improving on it is moot for scalar models, experience with renormalization of massless graphs suggests a less restrictive criterion. To wit, extension of log-homogeneous graphs in the sense of [3, 4] produces log-homogeneous graphs of the same order and higher degree. Whenever the extension can be made to a log-homogeneous graph of the same degree, we understand that we deal with a matter of (re)definition, the diagram is convergent, and no renormalization has taken place.

The subject is discussed in [3], but we deem it worthwhile to bring it here for completeness in our review of [2]. We show a truly trivial example in the Minkowski space $M_{4}$. The distribution $\delta\left(x^{2}\right)$ appears routinely in formulas for the propagators of free massless fields. However, its meaning is not altogether obvious. Given any distribution $f$ on $\mathbb{R}$, one is able to define pullback distributions $f(P)$ on smooth hypersurfaces $P$ (codimension 1 submanifolds) of $M_{4}$; in particular one defines $\delta(P)$. However the lightcone $x^{2}=0$ is not smooth at the origin, where $\operatorname{grad} x^{2}$ vanishes. Thus prima $f a$ cie $\delta\left(x^{2}\right)$ is defined on $M_{4} \backslash\{0\}$ only - as a homogeneous distribution of order -2 . Consider, however, for small positive $\varepsilon$, the distributions:

$$
\left\langle\delta_{ \pm}\left(x^{2}-\varepsilon\right), \phi\right\rangle=\int_{ \pm} \phi \mu_{\varepsilon}
$$

where the integrals are respectively concentrated on the upper and lower sheet of the hyperboloid $\left\{x: x^{2}=\varepsilon\right\}$, and $\mu_{\varepsilon}$ is a Leray form such that $d x^{2} \wedge \mu_{\varepsilon}=d^{4} x$. One can take $\mu_{\varepsilon}=\frac{d^{3} \boldsymbol{x}}{2 t(r ; \varepsilon)}:=\frac{d^{3} \boldsymbol{x}}{2 \sqrt{r^{2}+\varepsilon}}$ with $r \equiv|\boldsymbol{x}|$. Hence,

$$
\left\langle\delta_{ \pm}\left(x^{2}-\varepsilon\right), \phi\right\rangle=\frac{1}{2} \int \frac{\phi\left( \pm \sqrt{r^{2}+\varepsilon}, \boldsymbol{x}\right)}{\sqrt{r^{2}+\varepsilon}} d^{3} \boldsymbol{x}=2 \pi \int_{0}^{\infty} \frac{\bar{\phi}\left( \pm \sqrt{r^{2}+\varepsilon}, r\right)}{\sqrt{r^{2}+\varepsilon}} r^{2} d r .
$$

We have called $\bar{\phi}(t, r)$ the average value of $\phi$ on a sphere of radius $r$ in $\boldsymbol{x}$-space. Then the limits as $\varepsilon \downarrow 0$,

$$
\left\langle\delta_{ \pm}\left(x^{2}\right), \phi\right\rangle=2 \pi \int_{0}^{\infty} \bar{\phi}( \pm r, r) r d r
$$

are obviously well defined, and they extend the previous $\delta\left(x^{2}\right)$. 
The extension of $\delta\left(x^{2}\right)$ of course is not unique; but crucially the one just defined preserves Lorentz symmetry and keeps the same degree of homogeneity: we then reckon that no "renormalization" has taken place 1

A more sophisticated example is found in [3, Sect. 5]. The propagator of the free electromagnetic field is the quotient of a harmonic polynomial of degree 2 by the third power of $x^{2}$, and thus is logarithmically divergent by power counting. However, it does possess an homogeneous extension, therefore it is convergent in our more precise sense. The example offers little doubt, since, as is well known, it boils down to second derivatives of the massless propagator [9].

\section{Dealing with a three-point problem}

Let us continue by pointing to another difference between DiffRen as practiced in 1,2] and the (improved) Epstein-Glaser method 10. In the former papers one finds as extension for the "fish" graph of the $\phi_{4}^{4}$ model, with vertices $(0, x)$ :

$$
r_{x}\left[x^{-4}\right]=-\frac{1}{2} \Delta\left(x^{-2} \log \frac{|x|}{l}\right),
$$

in the understanding that $l=1 / M$, where $M$ is their mass scale; while, as explained in 4, we prefer

$$
R_{x}\left[x^{-4}\right]=-\frac{1}{2}\left[\Delta\left(x^{-2} \log \frac{|x|}{l}\right)-\pi^{2} \delta(x)\right],
$$

because $x^{2} R_{x}\left[x^{-4}\right]=x^{-2}$, while $x^{2} r_{x}\left[x^{-4}\right]$ fails to reproduce the convergent amplitude $x^{-2}$. The algebra property of [10] generalizes throughout into the "causal factorization property" of [3], which is the basis for a streamlined proof of the recursive renormalization of subdivergences.

Now, our lodestone to deal with recursive renormalization in [4] was a rule contained in the very illuminating paper [1]. It is written:

$$
\langle R[\Gamma], \varphi\rangle=\langle R[\gamma],(\Gamma / \gamma) \varphi\rangle .
$$

In this formula any subgraph $\gamma$ of a given graph $\Gamma$ is identified as a subset of the set of vertices of $\Gamma$ and the set of all lines joining any two elements of this subset. There $R[\Gamma], R[\gamma]$ and $\Gamma / \gamma$ denote amplitudes, respectively for the renormalized graph and subgraph, and the bare cograph $\Gamma / \gamma$; the test function $\varphi$ is supported outside the singular points of the latter. The rule works as a necessary and sufficient prescription: the $R[\gamma]$ are supposed known, and then $R[\Gamma]$ must conform to the formula above. It subsumes (the Euclidean version of) the causal factorization property, which allowed a streamlined proof of recursive renormalization in [3], but would be awkward in actual computation. Later we will show how rule (2) works when there are internal vertices in $\Gamma$, by means of an interesting six-loop diagram considered in 2 .

\footnotetext{
${ }^{1}$ The "original $\sin$ " still shows in that, whereas for $\varepsilon>0$ we can indefinitely apply to $\delta_{ \pm}\left(x^{2}-\varepsilon\right)$ the usual derivation rules, the expression $\delta^{\prime}\left(x^{2}\right)$ on $M_{4}$ is meaningless.
} 
In [2], on the other hand, it is claimed that recursive renormalization is effected by use of Bogoliubov's subtraction operators [12, Ch. 29]; another good reference for this method is [8]. For instance, according to them, the bare graph $x^{-4}$, with vertices $x, 0$, is renormalized by

$$
r_{x, 0}\left[x^{-4}\right]=\left(I-T_{x, 0}\right) x^{-4} \quad \text { with } \quad T_{x, 0} x^{-4}:=x^{-4}-\left(-\frac{1}{2} \Delta\left(x^{-2} \log \frac{|x|}{l}\right)\right) .
$$

Actually $T_{x, 0} x^{-4}$ as written above makes no sense, since if we compare both its summands on the intersection of their natural domains we obtain zero. But it is obvious how to get rid notationally of this ugly contortion, which makes violence to DiffRen.

Let us start in earnest by considering, as the authors in that paper do, the example of the winecup graph or ice-cream ladder graph, devoid of internal vertices:

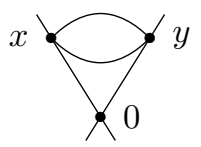

(In order to get closer to the notation in [2], we have exchanged vertices $x$

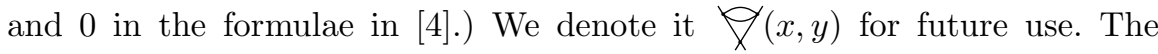
corresponding bare amplitude is given by

$$
f(x, y)=\frac{1}{x^{2} y^{2}(x-y)^{4}} .
$$

Both papers [1] and 4] consider a "partially renormalized" version of the winecup graph, for which the known formulas respectively yield:

$$
\begin{aligned}
r_{x, y}\left[x^{-2} y^{-2}(x-y)^{-4}\right]= & -\frac{1}{2} x^{-2} y^{-2} \Delta\left((x-y)^{-2} \log \frac{|x-y|}{l}\right) \\
R_{x, y}\left[x^{-2} y^{-2}(x-y)^{-4}\right]= & -\frac{1}{2} x^{-2} y^{-2} \Delta\left((x-y)^{-2} \log \frac{|x-y|}{l}\right) \\
& +\pi^{2} x^{-4} \delta(x-y) .
\end{aligned}
$$

The last expressions indeed make sense for all $(x, y) \neq(0,0)$.

The first term on the right hand sides above is the one given in 2 , Eq. 2.15]. Extension of the last term, not present there, to the thin diagonal (i.e., the whole graph), clearly is no problem. So we concentrate in extending the first: each of the factors in $r_{x, y}\left[x^{-2} y^{-2}(x-y)^{-4}\right]$ is a well-defined distribution, but their product is not.

The tactic followed in [1, 4, is to invoke Green's integration-by-parts formula to shift the Laplacian to the left and use the fundamental solution for it. Paper 2 purports instead to deal with the overall divergence "as a threepoint problem". Their claim is firstly that the whole graph is renormalized by

$$
\left(I-T_{x, y, 0}\right)\left(I-T_{x, 0}\right) x^{-2} y^{-2}(x-y)^{-4} .
$$

Secondly, that the handy and correct formula

$$
A(\Delta B)=(\Delta A) B+\partial_{\beta}\left(A \partial_{\beta} B-B \partial_{\beta} A\right),
$$


employed in 11 and borrowed by 4, is not to be used, on the grounds that such a trick reverts to a two-point problem, while they want to grapple directly with the three-point problem. Thirdly, that the singular behaviors of $r_{x, y}\left[x^{-2} y^{-2}(x-y)^{-4}\right]$ and of $-2 \pi^{2} \delta(y) x^{-4} \log |x| / l$ are the same.

Again, the mathematical argument there given for all that is hard to bear. However, the last assertion is correct, and it can be made sense of as follows. Note that

$$
\begin{aligned}
\left\langle x^{-2} z^{-2} \Delta\left((x-z)^{-2} \log \frac{|x-z|}{l}\right), \varphi(x, z)\right\rangle \\
=\left\langle x^{-2} z^{-2} \Delta\left((x-z)^{-2} \log \frac{|x-z|}{l}\right), \varphi(x, 0)\right\rangle \\
\quad+\left\langle x^{-2} y^{-2} \Delta\left((x-y)^{-2} \log \frac{|x-y|}{l}\right), \varphi(x, y)-\varphi(x, 0)\right\rangle .
\end{aligned}
$$

The second integral is finite, and the first one is proportional to

$$
\left\langle\delta(y) x^{-4} \log |x| / l, \varphi(x, y)\right\rangle .
$$

In practice we are back to the two-point problem. A similar argument works whenever the graph has been rendered "primitive" by partial renormalization. The authors of [2] continue their exposition as follows. In DiffRen one has

$$
r_{x, 0}\left[x^{-4} \log |x| / l\right]=-\frac{1}{4} \Delta \frac{\log ^{2}|x| / l+\log |x| / l}{x^{2}} .
$$

Therefore they write:

$$
\begin{gathered}
r_{x, y, 0}\left[r_{x, 0}\left[x^{-2} y^{-2}(x-y)^{-4}\right]\right]=-\frac{1}{2} x^{-2} y^{-2} \Delta\left((x-y)^{-2} \log |x-y| / l\right) \\
-2 \pi^{2} \delta(y) x^{-4} \log |x| / l-\frac{\pi^{2}}{2} \delta(y) \Delta \frac{\log ^{2}|x| / l+\log |x| / l}{x^{2}},
\end{gathered}
$$

with the contention that, although neither of the first two terms in the last expression exists strictu sensu, their combination is a well-defined distribution. The situation is then clarified by use of (44) nevertheless, whereby the second term in the above display completely drops out, and we are left with the third one plus a well-defined divergence - in the sense of vector calculus, see right below.

With our method, there comes

$$
\begin{aligned}
& -\frac{1}{2} x^{-2} y^{-2} \Delta_{y}\left((x-y)^{-2} \log \frac{|x-y|}{l}\right) \\
& =2 \pi^{2} \delta(y) x^{-4} \log \frac{|x|}{l}+\frac{1}{2} x^{-2} \partial_{y}^{\beta} L_{\beta}(x-y ; y)
\end{aligned}
$$

where

$$
L_{\beta}(x-y ; y):=(x-y)^{-2} \log \frac{|x-y|}{l} \partial_{\beta}^{y} y^{-2}-y^{-2} \partial_{\beta}^{y}\left((x-y)^{-2} \log \frac{|x-y|}{l}\right) .
$$


Moreover, the term $2 \pi^{2} \delta(y) x^{-4} \log |x| / l$ in our treatment, as done already in (3), is renormalized according to [4, Eq. A.4]:

$2 \pi^{2} R_{x, y, 0}\left[x^{-4} \log |x| / l \delta(y)\right]=-\frac{\pi^{2}}{2} \delta(y) \Delta \frac{\log ^{2}|x| / l+\log |x| / l}{x^{2}}+\pi^{4} \delta(x) \delta(y)$.

Finally, we ought to contend with the last term in (3):

$$
R_{x, y, 0}\left[\pi^{2} x^{-4} \delta(x-y)\right]=-\frac{\pi^{2}}{2} \Delta \frac{\log |x| / l}{x^{2}} \delta(x-y)+\pi^{4} \delta(x) \delta(y) .
$$

In summary, with obvious abbreviated notations:

$$
\begin{aligned}
& R_{x, y, 0} \nabla(x, y)=\frac{1}{2} x^{-2} \partial_{y}^{\beta} L_{\beta}(x-y ; y)-\frac{\pi^{2}}{2} \delta(y) \Delta \frac{\log ^{2}|x| / l+\log |x| / l}{x^{2}} \\
& -\frac{\pi^{2}}{2} \Delta \frac{\log |x| / l}{x^{2}} \delta(x-y)+2 \pi^{4} \delta(x) \delta(y) ; \\
& r_{x, y, 0} \mathcal{X}(x, y)=\frac{1}{2} x^{-2} \partial_{y}^{\beta} L_{\beta}(x-y ; y)-\frac{\pi^{2}}{2} \delta(y) \Delta \frac{\log ^{2}|x| / l+\log |x| / l}{x^{2}} .
\end{aligned}
$$

We remark that the differences between DiffRen and the improved EpsteinGlaser method are of no consequence for the $\beta$-function, up to third order in the coupling constant [4, Sect. 6].

Note that we may as well symmetrize:

$$
\begin{aligned}
r_{x, y, 0} \bigotimes(x, y)= & \frac{1}{4} x^{-2} \partial_{y}^{\beta} L_{\beta}(x-y ; y)-\frac{\pi^{2}}{4} \delta(y) \Delta \frac{\log ^{2}|x| / l+\log |x| / l}{x^{2}} \\
& +\frac{1}{4} y^{-2} \partial_{y}^{\beta} L_{\beta}(x-y ; x)-\frac{\pi^{2}}{4} \delta(x) \Delta \frac{\log ^{2}|y| / l+\log |y| / l}{y^{2}} .
\end{aligned}
$$

\section{More general procedures}

It is plausibly claimed in 2] that more complicated graphs can be tackled by the systematics of the Bogoliubov recursion, or its descendant the forest formula. That is,

$$
R \Gamma=\left(I-T_{\Gamma}\right) \bar{R}_{\Gamma},
$$

where the $\bar{R}_{\Gamma}$ object, renormalized but for the overall divergence is given by:

$$
\bar{R}_{\Gamma}=I+\sum_{\mathcal{P}} \prod_{v \in \mathcal{P}}\left(-T_{v} \bar{R} v\right) \prod \text { prop }
$$

where the sum is over the partitions of $\Gamma$ into divergent generalized vertices $v$, and $\prod$ prop denotes the product of propagators corresponding to all lines which connect the different elements of the partition.

The notational contortions above are again clear, but we do not take issue with them; rather we hasten to revisit a definitely amusing example. We ponder the six-loop graph $\Gamma$ obtained when one "stye" develops on each 
of the single propagators in a winecup graph:

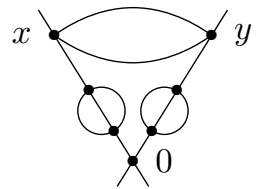

Our authors claim that, after dealing with the subdivergences, the partially renormalized graph comes out as

$$
r_{x, y} \Gamma \propto \frac{\log |x| / l}{x^{2}} \frac{\log |y| / l}{y^{2}} \Delta \frac{\log |x-y| / l}{(x-y)^{2}} ;
$$

and they set out to cure the overall divergence of the graph as $x, y \rightarrow 0$. Our method sustains their claim: each of the "dressed propagator" amplitudes for this diagram are of the form, with obvious labels:

$$
\iint r_{x, 0}\left[v^{-2}(v-w)^{-6}(w-x)^{-2}\right] d v d w=\iint r_{x, 0}\left[v^{-2} u^{-6}(v-u-x)^{-2}\right] d u d v .
$$

Notice that this is a nested convolution; the inner integral is of the form $R\left[r^{-6}\right] * r^{-2}$, which exists by the theory of [4, Sect. 3]. As long as convolution can be effected, rule (2) proceeds without obstruction.

The displayed integral becomes

$$
-\frac{1}{16} \iint v^{-2}(v-u-x)^{-2}\left(\Delta^{2}\left(u^{-2} \log \frac{|u|}{l}\right)-5 \pi^{2} \Delta \delta(u)\right) d v d u .
$$

On integrating by parts and dropping total derivatives in the integrals over internal vertices, we then obtain

$$
\begin{aligned}
& \frac{\pi^{2}}{4} \iint v^{-2}\left(\Delta\left(u^{-2} \log \frac{|u|}{l}\right)-5 \pi^{2} \delta(u)\right) \delta(v-u-x) d v d u \\
& \quad=\frac{\pi^{2}}{4} \int(u+x)^{-2}\left(\Delta\left(u^{-2} \log \frac{|u|}{l}\right)-5 \pi^{2} \delta(u)\right) d u \\
& \quad=-\pi^{4} \int u^{-2} \log \frac{|u|}{l} \delta(u+x) d u-\frac{5 \pi^{4}}{4} x^{-2}=-\pi^{4} x^{-2}\left(\log \frac{|x|}{l}+\frac{5}{4}\right),
\end{aligned}
$$

where the extra term with respect to formula (17) is due to our different treatment of the basic "sunset" self-energy diagram: as usual we define it so that

$$
x^{2} R_{x, 0}\left[x^{-6}\right]=R_{x, 0}\left[x^{-4}\right]
$$

which fails for DiffRen.

Therefore we have to renormalize the overall divergence

$$
\begin{gathered}
\pi^{8}\left(\frac{\log |x| / l}{x^{2}} \frac{\log |y| / l}{y^{2}}+\frac{5}{4} x^{-2} \frac{\log |y| / l}{y^{2}}+\frac{5}{4} y^{-2} \frac{\log |x| / l}{x^{2}}+\frac{25}{16} x^{-2} y^{-2}\right) \\
\times\left(-\frac{1}{2} \Delta\left((x-y)^{-2} \log \frac{|x-y|}{l}\right)+\pi^{2} \delta(x-y)\right) .
\end{gathered}
$$


Several of the terms above bring nothing new; we concentrate on the most difficult one, of the form (77), the only one recognized in [2]. Its authors argue that as $x \sim y \sim 0$ one has:

$$
r_{x, y} \Gamma \sim-32 \pi^{2} \delta(y) \frac{\log ^{3}|x| / l}{x^{4}},
$$

which they renormalize en DiffRen as

$$
4 \pi^{2} \delta(y) \Delta \frac{\log ^{4}|x| / l+2 \log ^{3}|x| / l+3 \log ^{2}|x| / l+3 \log |x| / l}{x^{2}} .
$$

We pause to point to our essentially coincident formula [4, A.2]:

$$
\begin{aligned}
R_{x, 0} & \frac{\log ^{3}|x| / l}{x^{4}} \\
& =-\frac{1}{8} \Delta \frac{\log ^{4}|x| / l+2 \log ^{3}|x| / l+3 \log ^{2}|x| / l+3 \log |x| / l}{x^{2}}+\frac{3}{4} \pi^{2} \delta(x) .
\end{aligned}
$$

Of course, the expression resulting from (8) and (9):

$$
\begin{aligned}
& \frac{\log |x| / l}{x^{2}} \frac{\log |y| / l}{y^{2}} \Delta \frac{\log |x-y| / l}{(x-y)^{2}}+4 \pi^{2} \delta(y)\left[\frac{8 \log ^{3}|x| / l}{x^{4}}\right. \\
& \left.+\Delta \frac{\log ^{4}|x| / l+2 \log ^{3}|x| / l+3 \log ^{2}|x| / l+3 \log |x| / l}{x^{2}}\right]
\end{aligned}
$$

is rather ugly, since the first two terms are undefined. However, they cleverly add and subtract to it $y^{-2} x^{-2} \log |x| / l \Delta\left((x-y)^{-2} \log |x-y| / l\right)$, and applying Green's formula, everything is rewritten:

$$
\begin{aligned}
& \frac{\log |x| / l}{x^{2}} \frac{\log |y| / l}{y^{2}} \Delta \frac{\log |x-y| / l}{(x-y)^{2}} \longmapsto \frac{\log |x| / l}{x^{2}} \frac{\log |y| /|x|}{y^{2}} \Delta \frac{\log |x-y| / l}{(x-y)^{2}} \\
& +\frac{\pi^{2}}{2} \delta(y)\left[\Delta \frac{\log ^{4}|x| / l+2 \log ^{3}|x| / l+3 \log ^{2}|x| / l+3 \log |x| / l}{x^{2}}\right] \\
& \quad-\frac{\log ^{2}|x| / l}{x^{2}} \partial_{y}^{\beta} L_{\beta}(x-y ; y) .
\end{aligned}
$$

The way to improve on this is symmetrization:

$$
\begin{gathered}
r_{x, y, 0} r_{x, y} \Gamma=\frac{\pi^{2}}{2} \delta(y)\left[\Delta \frac{\log ^{4}|x| / l+2 \log ^{3}|x| / l+3 \log ^{2}|x| / l+3 \log |x| / l}{x^{2}}\right] \\
+\frac{\pi^{2}}{2} \delta(x)\left[\Delta \frac{\log ^{4}|y| / l+2 \log ^{3}|y| / l+3 \log ^{2}|y| / l+3 \log |y| / l}{y^{2}}\right] \\
-\frac{\log ^{2}|x| / l}{x^{2}} \partial_{y}^{\beta} L_{\beta}(x-y ; y)-\frac{\log ^{2}|y| / l}{y^{2}} \partial_{x}^{\beta} L_{\beta}(y-x ; x) .
\end{gathered}
$$

The task of computing the remaining terms in $R_{x, y, 0} R_{x, y} \Gamma$ is comparatively easier. The ones containing the factor $\delta(x-y)$ simply go into

$$
\pi^{10} \delta(x-y)\left[R_{x, 0} \frac{\log ^{2}|x| / l}{x^{4}}+\frac{5}{2} R_{x, 0} \frac{\log |x| / l}{x^{4}}+\frac{25}{16} R_{x, 0} \frac{1}{x^{4}}\right]
$$


in terms of known renormalized expressions [4]. Of the three remaining terms, two are totally similar:

$$
-\frac{5}{8}\left(x^{-2} \frac{\log |y| / l}{y^{2}}+y^{-2} \frac{\log |x| / l}{x^{2}}\right) \Delta\left((x-y)^{-2} \log \frac{|x-y|}{l}\right),
$$

and can be computed as above by Green's formula, and the other is of the same form as the winecup graph.

\section{Conclusion}

Formulae (5) and (6) work like guiding principles, rather than calculational recipes. Actual production of closed formulas relies on a bag of tricks. While the application of the inductive principle of 4 often profits from similar tricks, it appears to be better adapted in practice to deal with complex diagrams.

\section{Acknowledgments}

The author is thankful to Joseph C. Várilly for some very useful comments. His work was supported by the Spanish Ministry for Science through grant FPA2012-35453.

\section{References}

[1] D. Z. Freedman, K. Johnson and J. I. Latorre, Differential regularization and renormalization: a new method of calculation in quantum field theory. Nucl. Phys. B 371 (1992), 352-414.

[2] J. I. Latorre, C. Manuel and X. Vilasís-Cardona, Systematic differential renormalization at all orders. Ann. Phys. 231 (1994), 149-173.

[3] N. M. Nikolov, R. Stora and I. Todorov, Renormalization of massless Feynman amplitudes in configuration space. Rev. Math. Phys. 26 (2014), 1430002.

[4] J. M. Gracia-Bondía, H. Gutiérrez and J. C. Várilly, Improved Epstein-Glaser renormalization in $x$-space versus differential renormalization. Nucl. Phys. B 886 (2014), 824-869.

[5] M. Dütsch, K. Fredenhagen, K. J. Keller and K. Rejzner, Dimensional regularization in position space, and a forest formula for Epstein-Glaser renormalization. J. Math. Phys. 55 (2014), 122303.

[6] H. Epstein and V. Glaser, The role of locality in perturbation theory. Ann. Inst. Henri Poincaré A 19 (1973), 211-295.

[7] S. Weinberg, High-energy behavior in quantum field theory. Phys. Rev. 118 (1980), 838-849.

[8] E. B. Manoukian, Renormalization, Academic Press, London, 1983.

[9] R. Stora, in Fifty years of Yang-Mills theory, G. 't Hooft, ed., World Scientific, Singapore, 2005; pp. 137-167.

[10] J. M. Gracia-Bondía, Improved Epstein-Glaser renormalization in coordinate space I. Euclidean framework. Math. Phys. Anal. Geom. 6 (2003), 59-88. 
[11] A. N. Kuznetsov, F. V. Tkachov and V. V. Vlasov, Techniques of distributions in perturbative quantum field theory I. arXiv:hep-th/9612037.

[12] N. N. Bogoliubov and D. V. Shirkov, Introduction to the theory of quantized fields. 3rd Edition, Wiley, New York, 1980.

José M. Gracia-Bondía

Department of Theoretical Physics

Universidad de Zaragoza

50009 Zaragoza, Spain;

BIFI Research Center

Universidad de Zaragoza

50018 Zaragoza, Spain;

Institute for Theoretical Physics

Universidad Autónoma

28049 Madrid, Spain. 